\title{
A Case Report of A Patient of Iga Nephropathy Associated With Psoriasis: A Contribution To The Entity of 'Psoriatic Nephropathy'
}

\author{
Dr. Bharat Veer Manchanda ${ }^{1}$, Dr. Gaurav Aggarwal ${ }^{2}$, Dr. Mini Bhatnagar ${ }^{3}$, \\ Dr. Jatin Aggarwal ${ }^{4}$, Dr. Saloni Mehra ${ }^{5}$, Dr. Neeraj Gupta ${ }^{6}$ \\ ${ }^{1}$ Assi. Professor In Dept Of Medicine, Subharti Medical College, Meerut \\ ${ }^{2,6}$ Post Graduate In Dept Of Medicine, M.M.I.M.S.R, Mullana. \\ ${ }^{3}$ Asso. Professor In Dept Of Medicine, M.M.I.M.S.R, Mullana. \\ ${ }^{4}$ Post Graduate In Dept Of Orthopedic, M.M.I.M.S.R, Mullana \\ ${ }^{5}$ Post Graduate In Dept Of Surgery, Subharti Medical College, Meerut
}

\begin{abstract}
Occurrence of glomerular disease in psoriasis is rare, though the number of case reports is increasing in the recent years. Different types of glomerular involvement have been reported but mesangioproliferative glomerulonephritis with IgA deposits is common in association with psoriasis. The term 'psoriatic nephropathy' has been introduced recently. We contribute a case to the on-going discussion regarding psoriatic nephropathy. Our patient had mesangio-proliferative glomerulonephritis with $\operatorname{IgA}(3+), \operatorname{Ig} G(1+) \operatorname{IgM}(2+)$ and $\mathrm{C} 3(2+)$ in association with psoriasis. The patient was managed on maintenance haemodialysis and immunosuppressive therapy (cyclophosphamide).
\end{abstract}

\section{Introduction}

Psoriasis is an immune mediated chronic inflammatory disorder of the skin with distinct micro vascular changes.It can involve joints but involvement of internal organs is uncommon. Psoriatic nephropathy is a recently described clinical disorder. Nephropathy in association with psoriasis is increasingly being reported in recent years.

\section{Casereport}

43 years old male from Saharanpur with wide spread psoriasiswith hair and nail involvement from 6-7 years not on regular treatment presented with swelling of the face and both lower limbs for last 10 days, along with breathlessness and decreased urine output for the same duration. No H/o joint pain, swelling, haematuria. No previous H/O Diabetes mellitus,tuberculosis,hypertension.

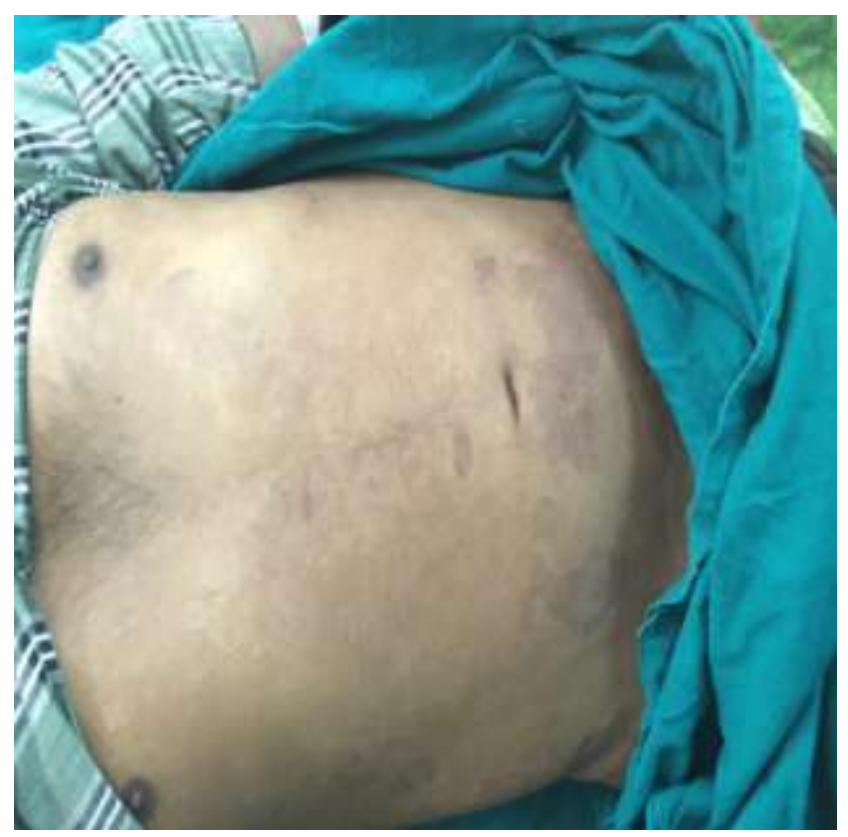

Figure 1 psoriasis with involvement of trunk 


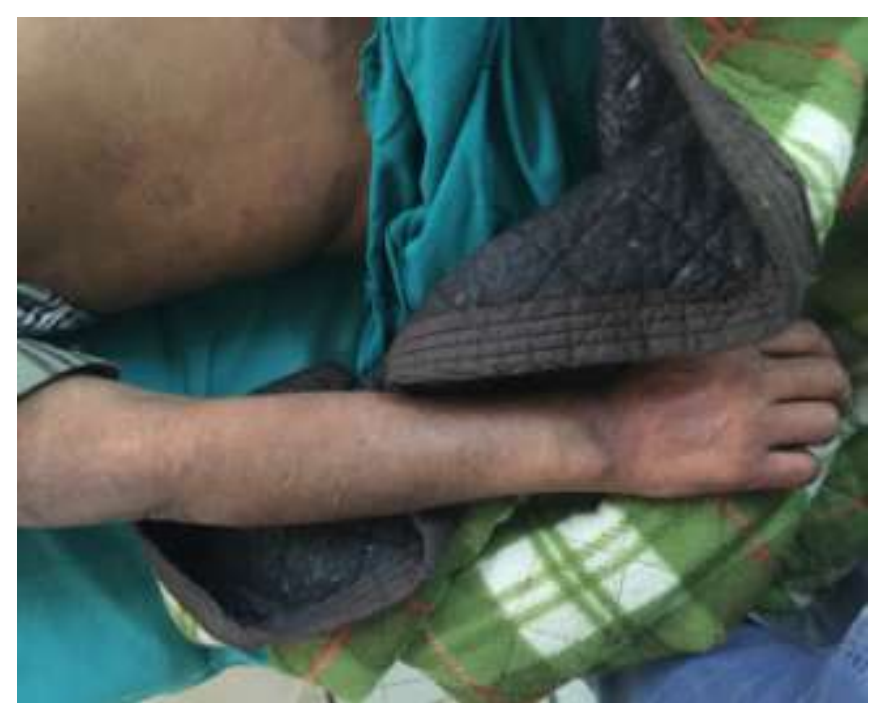

Figure 2 psoriasis on extensor surface of the forearm

On examination patient was conscious, oriented and hypertensive. His BP was 170/90mmhg.The patient had puffiness of face, pedal edema.He had wide spread skin lesions distributed over both upper and lower extremities as well as on the trunk(fig 1 and 2). Nail pitting was also present. He was already diagnosed case of psoriasis vulgaris.There was no organomegaly.Examination of the other systems revealed no abnormality.

His blood picture revealed $\mathrm{Hb} 10.4$ gm\%,TLC -8000/cumm with neutrophill71\%,lymphocytes $25 \%$ eosinophills $03 \%$ monocytes $1 \%$ and ESR $60 \mathrm{~mm} / \mathrm{hr}$.LFT were within normal limits with Urea of 134 and creatnine $12.19 \mathrm{mg} / \mathrm{dl}$. serum protein was 5.8 and albumin $1.5 \mathrm{gm} / \mathrm{dl}$ with lipid profile within normal limits. Urine examination revealed albumin+++,rbc+,epithelial cells (10-12) sugar-nil with casts+, with 24 hours urinary protein of $1360 \mathrm{mg}$. Kidney biopsy shows 11 glomeruli, 4 of which are sclerosed 2 glomeruli show fibro cellular crescents and 2 shows fibrous crescents and 2 shows segmental sclerosis (diffuse mesangio proliferative glomerulonephritis) with immunofluorescence study shows $\operatorname{IgA}(3+), \operatorname{IgG}(1+) \operatorname{IgM}(2+)$ and $\mathrm{C} 3(2+)$ deposition. ELISA for HIV.HBsAg and anti-HCV were non-reactive.

The patient was treated with topical clobetasol ,salicylate ointment,coconut oil. Renal problem was treated with diuretics,antihypertensive and haemodialysis and immunosuppressive therapy with cyclophosphamide. Nutritional support including multivitamin and minerals were added to improve immune mechanism. Oedema subsided,RFT improved and blood pressure came to normal with urine output remains the same, with patient discharged under stable conditions with further follow up.

\section{Discussion}

Immunoglobulin A nephropathy is characterised by prominent diffuse mesangial IgA deposits at immunofluorescent microscopy. The condition was initially thought to be a rare and benign cause of recurrent hematuria. IgAN has been associated with several diseases, such as celiac sprue, seronegativespondyloarthropathies, posinfectious arthritis, ulcerative colitis, regional enteritis, dermatitis herpetiformis, malignancies, mixed cryoglobulinemia, human immunodeficiency virus (HIV) infection and idiopathic pulmonary hemosiderosis (1). Its association with psoriasis has been rarely described. Singh et al (2) observed three patients with psoriasis and glomerulonephritis. Psoriasis was accompanied by IgA nephropathy in one of these cases, by focal proliferative glomerulonephritis in the second and by membranous glomerulopathy in the third. The majority of literature reports, mainly casuistic, refer to the coexistence of psoriasis and IgA nephropathy (3). Increasing reports of nephropathy in psoriasis are arguing against chance association. But the exact relationship of psoriasis and nephropathy remains unclear, but an autoimmune mechanism most likely links the two. Probably the same inflammatory mechanism of psoriatic arthritis is responsible for nephropathy. Drug related nephropathy in psoriasis should be kept in mind because of high usage of offending drugs (e.g., NSAIDs in psoriatic arthritis).

In our study, we report a patient with psoriasis who was then diagnosed with $\operatorname{IgA}$ nephropathy.. Patients with psoriasis might show varying degrees of glomerular involvement, presenting with microalbuminuria, hematuria, nephrotic syndrome and a deterioration in renal function(4) .The patient was treated with steroids,immunosupressive therapy and withhemodialysis and responded to the treatment. 


\section{Conclusion}

Psoriatic nepropathy is a recently described disorder. Routine urinalysis, kidney function assessment and a wider application of renal biopsy in psoriatic patients may be helpful to detect renal involvement (subclinical or overt nephropathy). Psoriatic nepropathy may respond to methotrexate like psoriatic arthritis. Large trials are needed to establish the role of methotrexate in psoriasis associated nephropathy.

\section{References}

[1]. Julian BA, Wyatt RJ, Matousovic K, Moldoveanu Z, Mestecky J, Novak J: IgA nephropathy: A clinical overview. ContribNephrol 2007; 157: 19-26

[2]. Singh NP, Prakash A, Kubba S, Ganguli A, Singh AK, Sikdar S, Agarwal SK, Dinda AK, Grover C: Psoriatic nephropathy does an entity exist? Ren Fail 2005; 27: 123-127

[3]. Yamamoto M, Yorioka T, Kawada M, Nishimura K, Kumon Y, Yasuoka N, Suehiro T, Hashimoto K: A case of IgA nephropathy associated with psoriasis vulgaris. Nippon JinzoGakkai Shi 1994; 36: 779-783

[4]. Sirolli V, Bonomini M: Glomerulopathies associated with psoriasis: A report of three cases. Nephron 2000; 86: 89-90 